\title{
Initial Observations of Psychological and Behavioral Effects of COVID-19 in the United States, Using Google Trends Data
}

\author{
Daniel Goldman
}

March 2020

\section{Introduction}

It can be difficult to study changes in psychology and behavior, for a large group of people, that might occur over short periods of time. Surveys can be helpful, but they can also be expensive and time consuming, especially if a large sample is preferred or required. Additionally, while the surveys may be able to capture monthly, or even weekly changes in states, it can be difficult to capture daily changes and identify trends that require a finer resolution than weekly data.

It would be useful to be able to investigate how peoples' lives have changed on a day to day basis. However, doing so requires access to high resolution data. Luckily, sociology, public health, and other areas of population study have new tools available, thanks to operations like Google, and specifically publicly accessible search trend information. While not a direct measure of either psychology or behavior, it offers inter and even intraday data on the terms for which people are searching.

The various shelter-in-place orders, and other changes to daily activities, associated with the recent COVID-19 outbreak in the United States has likely had a significant impact on the United States population, with changes to their daily lives. Not only is COVID-19 likely affecting stress levels, it is also definitely affecting peoples' routines. Google Trends data should be able to give us insight into general patterns of psychological states and behaviors, as well as specific effects resulting from COVID-19.

\section{Psychological Effects}

Google Trends is a powerful tool for population level psychological analysis. We can't easily poll a large portion of the population, day after day, and see how they are feeling. But we can look at search habits. The tool has been used in quite a bit of research. Both Nuti et al. 2014 and Seung-Pyo et al. 2018 analyze a number of studies that utilize Google Trends data[3, 2]. 
The former study looked at 70 studies conducted between 2009 and 2013 and suggested a seven fold increase during that time period. Of those studies, $24 \%$ covered topics of mental health and substance use. The latter analyzes 657 research papers. The research suggests that reliance on Google Trends increased substantially in the 10 years leading up to the study and that in recent years, trend data is used in combination with other big data sources.

Public health science is always difficult, because it involves so many different people, and the collection of significant amounts of data as a result. For epidemiology, we can often engage in broad surveillance, or at least rely on hospital test results. Analyzing group psychology can be a little more complicated, especially if we're trying to identify trends in psychological states, over short periods of time. This study utilizes Google Trends data to identify changing mental states

and patterns of behavior associated with the COVID-19 outbreak. Search terms analyzed include anxiety, depression, therapy, suicide, and boredom.

\subsection{Seasonality of Stress Related Searches}

One of the first observations when looking at the trends data is an apparent seasonality to search trends related to stress, such as anxiety and depression. This seasonality appears to have a length of roughly seven days, with peaks during the middle of the week and lows during the weekends. Trends data from September 1, 2019 through February 29, 2020 was used for the initial analysis. This time period gives a large sample of data, within a time period leading up to, but not including the time when social distancing guidelines were in effect. Four search terms were chosen: anxiety, depression, therapy, and suicide.

To test for actual seasonality, vs subjectively identified seasonality a simple autocorrelation analysis was conducted. Analysis was conducted visually, using the plot_acf function in version 0.11.1 of the Python module, statsmodels. As expected, significant autocorrelation was found around a time shift of seven days, for the terms anxiety, depression, and therapy. However no such autocorrelation was found for suicide. This autocorrelation may be indicative increased stress during the workweek and greater time available to engage in stress reducing activities during the weekend. This analysis is not the first to identify a seven day seasonality to certain search terms. The seven day seasonality of searches for depression was identified as far back as 2008[1].

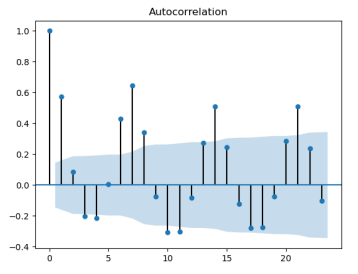

Figure 1: Autocorrelation Function for "Anxiety" 


\subsection{Changing Stress Related Searches}

The abrupt change in lifestyle caused by lockdowns across the United States has given us an interesting opportunity to investigate how search trends can change over short periods of time. All across the country, restaurants and bars are closing, or only offering takeout and delivery options, while a large number of people are being temporarily laid off. And the associated seven day cycle does appear to cease around the middle of March.

However, visual analysis again can be subjective. Change point detection is a statistically robust method for identifying changes in time series. In this case, binary segmentation analysis using version 1.0.3 of the ruptures Python package was selected. The normal model was chosen with four break points. For brevity, only the trends data for anxiety was analyzed in this way. Based on the analysis, there is a clear long term channel, along with break points identified on January 13, March 13, March 18, and March 23.

This change in search activity acts as a partial test for the cause of the seasonality in the data. This shift in trend makes sense. The stress from work is no longer present, but there are fewer stress reducing activities available as well. This lack of available activities would reasonably cause an increase in boredom.

\subsection{Boredom}

To test whether there was a change in searches for boredom, trends data for "boredom" was collected on April 3, and included the data from January 3, 2020 through April 1, 2020. Visually, the change in the rate of searching for "boredom" was obvious. Searches fluctuated over time, but roughly stayed within a channel. There was a stark increase around March 14. Between that date and March 20, searches for the term increased at a roughly linear rate, before stabilizing, resulting in a new channel that was roughly twice the level of the old channel. Again, change point analysis was conducted. The break points detected were on January 8, March 13, March 18, and March 23, which largely coincides with visual analysis. Moreover, three break points coincide with the change point analysis conducted on anxiety data.

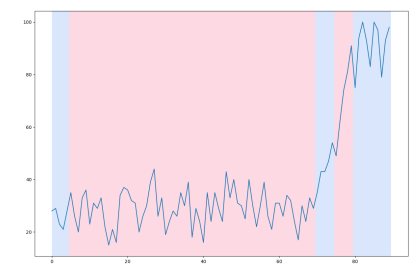

Figure 2: Change Point Analysis for Boredom 


\section{Changes in Food Related Activities}

Aside from changes in stress related search terms, it seemed reasonable that the change in work schedule and partial closing of restaurants would have an impact on food related searches. For constancy, the same time period was used for an initial analysis of trends as well as the secondary analysis for changes in trends. The search terms selected were cooking, baking, sourdough, and restaurant. All search terms are general, except for "sourdough." The sourdough search term was included for two reasons. For transparency, the author is likely somewhat biased regarding the topic, because of their interest in the baking sourdough bread. However, sourdough can be made without the need for commercial yeast. The lockdowns across the country, and the fear associated with the COVID-19 epidemic, have reduced access to a variety of ingredients, including yeast, and many individuals might be concerned that they will not be able to have access to yeast when they want to bake.

\subsection{Seasonality}

As was the case with stress related terms, there does appear to be a seasonality with food related terms, though the seasonality for cooking and baking do not appear to be as pronounced. The seasonality for restaurant searches is however striking, with negative correlation between weekdays and weekends, and high positive correlation for the seven day shift.

There also appeared to be some delay between searches for cooking and searches for restaurants. To test this visual analysis, and assuming that the relationship between the two were not linear, the Spearman's Rank correlation coefficient was calculated using Scipy package version 1.4.1, with positive and negative lags. As expected, the coefficient was much higher, and the p-value much lower, when the restaurant data was shifted one day forward. However, when doing the same analysis comparing cooking and baking and baking and restaurant searchers, zero lag gave the largest correlation and smallest p-value. For some reason, people appear to search for restaurants, before they search cooking. There relationship between searches for baking and searches for restaurants is less clear.

\begin{tabular}{||c|c|c||}
\hline & correlation & $\mathrm{p}$-value \\
\hline \hline Cooking (No-Shift) & 0.552 & $7.02 \times 10^{-16}$ \\
\hline Cooking (Shifted) & 0.637 & $3.85 \times 10^{-22}$ \\
\hline Baking/Cooking (No-Shift) & 0.826 & $1.28 \times 10^{-46}$ \\
\hline Baking/Cooking (Shifted) & 0.393 & $3.97 \times 10^{-08}$ \\
\hline Baking (No-Shift) & 0.511 & $1.77 \times 10^{-13}$ \\
\hline Baking (Shifted) & 0.492 & $1.66 \times 10^{-12}$ \\
\hline
\end{tabular}




\section{Shifting Behaviors}

Not surprisingly, there appears to be a significant falloff in searches for restaurants, and an increase in searches for cooking. Visually, the shift appears more pronounced in restaurant searches, likely because of all the restaurant closures. Meanwhile, people can still order takeout from places they already know about. Once again the same change point analysis technique was used to justify the visual analysis. Cooking was selected as the search term to analyze, in this instance. A primary channel was again found, with break points on January 28, March 18, March 23, and March 28. In this case, two break points are in common with the other analyses.

\section{Discussion}

Numerous changes in trends have been identified in this paper, and apparent points of change have been identified. In general, that March 18 and March 23 were common break points found in the analysis of different search trends is important. It helps justify the COVID-19 outbreak as a common thread. However, the identification of March 18 as an apparent break point does not mean that changes in psychology and behavior initiated on these dates.

Regarding the change in time series data identified when analyzing boredom, I expect that a new channel will indeed become apparent once the final data is analyzed. I also expect levels of stress to continue to increase before the lockdowns end, especially if they are extended past the April 30 goal.

Because this paper was written before the end of the lockdown, more data will become available over time, and further research can be conducted. In time, we will be able to use the full time period associated with the COVID-19 lockdown, in order to get a better picture of how the epidemic has impacted search trends, and we can further investigate the changes to peoples' lives caused by this unusual situation. While select terms were chosen for this study, Google Trends can offer insights into other changes in psychology associated with the COVID-19 outbreak, and further research should be done on these topics.

Meanwhile, the trend analysis for baseline data gives insight into seasonal trends for a variety of psychological states and various activities. The apparent increase in stress related searches during the middle of the week, and the potential relationship to actual increases in stress during that time period, should give the mental health and public health communities cause to research how we can reduce midweek stress. Moreover, the high granularity of this data may be useful in identifying whether such efforts are effective.

\section{References}

[1] Peter J. Ellery et al. "Understanding internet health search patterns: An early exploration into the usefulness of Google Trends". In: Journal of Com- 
munication in Healthcare 1.4 (Oct. 2008), pp. 441-456. DOI: 10.1179/cih . 2008.1.4.441. URL: https://doi.org/10.1179/cih.2008.1.4.441.

[2] Seung-Pyo Jun, Hyoung Sun Yoo, and San Choi. "Ten years of research change using Google Trends: From the perspective of big data utilizations and applications". In: Technological Forecasting and Social Change 130 (May 2018), pp. 69-87. DOI: 10.1016/j . techfore.2017.11.009. URL: https://doi.org/10.1016/j.techfore.2017.11.009.

[3] Sudhakar V. Nuti et al. "The Use of Google Trends in Health Care Research: A Systematic Review". In: PLoS ONE 9.10 (Oct. 2014). Ed. by Martin Voracek, e109583. DOI: 10 .1371/journal pone .0109583. URL: https://doi.org/10.1371/journal. pone.0109583. 